\title{
Geburtshaus Wilhelm Conrad Röntgen
}

\section{„Es ist das Gefühl eines besonderen Nucleus“}

Während die Tagungs- und Konferenzräumlichkeiten im Obergeschoss und ein Gäste- und Stipendiaten-Apartment im Dachgeschoss die fachwissenschaftliche Community adressieren, soll eine öffentliche und kostenfrei zugängliche Ausstellung im Erdgeschoss zu einem Magneten für die breite Öffentlichkeit werden. Für die Konzeption und Umsetzung konnte mit Dr. Ulrich Hermanns ein äußerst profilierter Ausstellungsmacher gewonnen werden. Im Interview gibt er einen Einblick in die zukünftige Ausgestaltung der historischen Räumlichkeiten.

Herr Dr. Hermanns, Ihr Büro Dr. Ulrich Hermanns Ausstellung Medien Transfer $\mathbf{G m b H}$ wurde im Frühjahr 2017 mit der Konzeption, Planung und Umsetzung einer Publikumsausstellung im Röntgen-Geburtshaus beauftragt. Doch Ihre Beziehung zu diesem Erinnerungsort hat bereits eine längere Vorgeschichte, oder?

Das stimmt, wir haben bereits 2014 ein erstes Konzept für die Nutzung und Ausstattung des Hauses erarbeitet, mit dem zugleich Unterstützer, Förderer und Sponsoren angesprochen werden sollten. Gemeinsam mit der Deutschen Röntgengesellschaft und dem Deutschen RöntgenMuseum entstanden eine Broschüre und ein Film, mit denen die künftige Nutzung und die Angebote des Hauses anschaulich wurden.

Seit rund 20 Jahren konzipieren und realisieren Sie Ausstellungen und eröffnen neue Zugänge insbesondere zu kulturhistorischen Orten. Was macht aus Ihrer Sicht das Geburtshaus von Wilhelm Conrad Röntgen zu einem besonderen, vielleicht auch einzigartigen Ort?

Es ist das Gefühl eines besonderen Nucleus, wenn man das Haus sieht und betritt. Es liegt in der Altstadt von Remscheid/Lennep leicht erhöht, es ist ein Eckhaus, man geht von unten kommend darauf zu, und es zieht einen irgendwie magisch an. Es hat eine Ausstrahlung. Das setzt sich im Inneren fort. Auch wenn Röntgen hier nur eine kurze Zeit seines Lebens verbrachte, bleibt

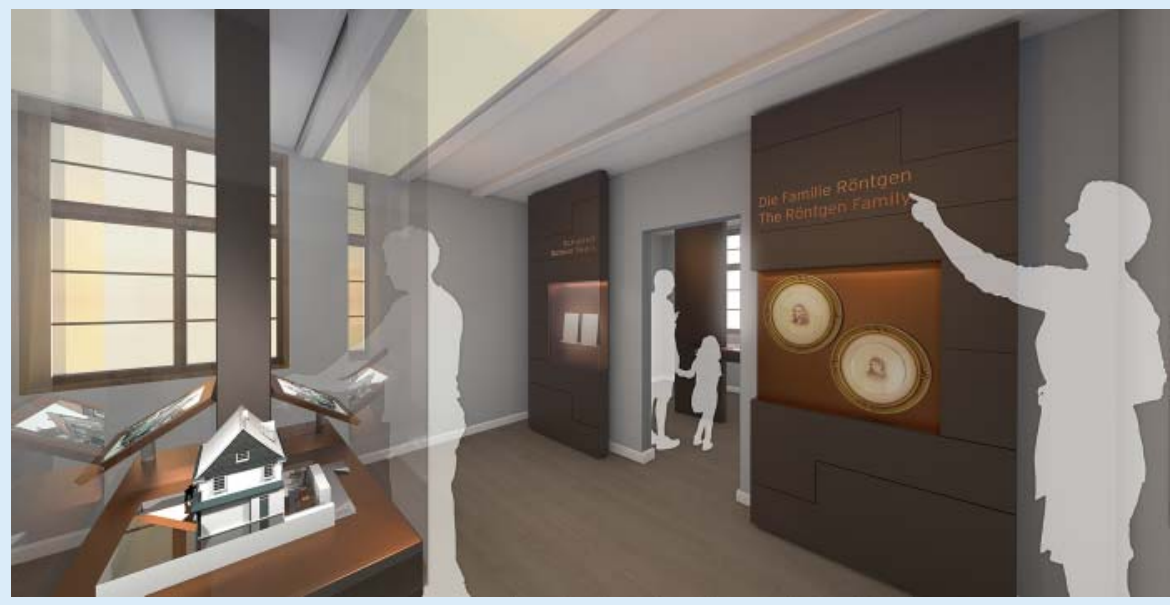

Blick in den Raum „Das Haus und die Familie“, (c) Dr. Ulrich Hermanns GmbH, Münster

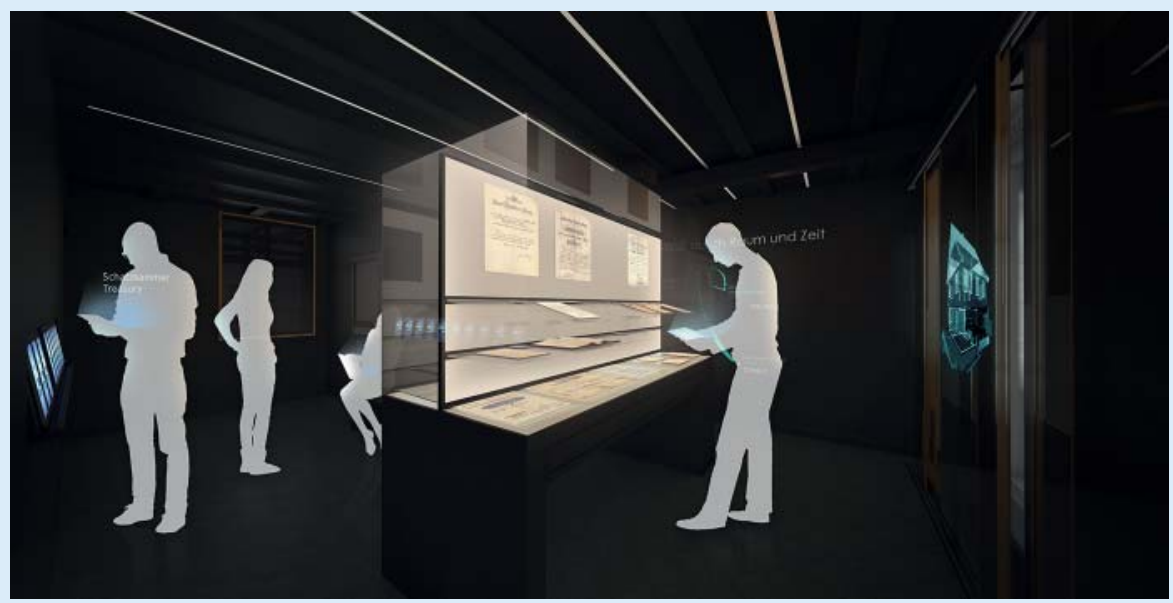

Blick in den Raum „Die Schatzkammer“, (c) Dr. Ulrich Hermanns GmbH, Münster

der Geburtsort doch für jeden Menschen immer etwas Einmaliges; und die Möglichkeit der künftigen Besucher, an diesem Ursprung teilzuhaben, ist ein besonderes Erlebnis.

Das Geburtshaus soll zukünftig ein aktiver Ort für Informationsvermittlung, gedanklichen Austausch und geistige Arbeit sein. Vor diesem Hintergrund nimmt die von Ihnen verantwortete Publikumsausstellung zur Person Röntgen im Erdgeschoss des Gebäudes einen besonderen Stellenwert ein. Welcher Leitgedanke bzw. welcher zentraler Ansatz liegt Ihrem Ausstellungskonzept zugrunde?
Das Konzept unterstützt diesen auch emotionalen Moment der Begegnung: Der Begegnung mit einer Biografie und Lebensleistung, die fast jeder Mensch auf der Welt zumindest durch den Vorgang des Röntgens selber schon erfahren hat. Der Begegnung mit dem Haus, der Person Wilhelm Conrad Röntgens und seiner Familie - und mit anderen Besuchern, die dorthin kommen, um sich in dieser speziellen Atmosphäre auszutauschen.

Wie haben Sie die Historie und Beschaffenheit der Ausstellungsräume, insbesondere aber deren verhältnismäßig geringe Grundfläche, konzeptionell verarbeitet? 
Das Konzept konzentriert sich auf wenige Interventionen in den Räumen. Sie widmen sich ganz der Person Röntgens, seiner Biografie und seinen Netzwerken, lassen aber den authentischen Räumen noch Luft zum Atmen. Technische Aspekte sind nicht das Thema, diese werden erlebnisreich im fußläufig entfernten Röntgen-Museum präsentiert. Die beiden Häuser ergänzen sich also in idealer Weise. Wichtig sind uns Aspekte der Barrierefreiheit, die in den kleinen Räumen eine Mobilität aber auch differenzierte kognitive Ansprache ermöglicht.

Welche Themen, Aspekte, Merkmale aus dem Leben und Wirken von Wilhelm Conrad Röntgen möchten Sie in der Ausstellung besonders hervorheben?

Wer war der Mensch Röntgen, wer waren seine Familie, Freunde, Kollegen und Bewunderer? Wir erschließen den Besuchern das weltweite Netzwerk, das er zu Lebzeiten pflegte. Die Besucher treffen hier in Lennep die führenden Denker der damaligen Zeit - in einer Nussschale sozusagen. Und dies anhand ausgewählter Originale, die das Archiv des Museums wie einen Schatz hütet, aber für diese Ausstellung dankenswerterweise zur Verfügung stellt. Dafür müssen spezielle konservatorische und sicherheitsrelevante Vorkehrungen getroffen werden.

Haben Sie für sich bereits ein besonderes Highlight ausgemacht, etwas, worauf Sie sich in der Ausstellung besonders freuen?

Ich finde die Briefe sehr spannend, die ihn aus unterschiedlichen Beweggründen und von verschiedenen Seiten erreichten. Es haben sich nicht sehr viele erhalten, da sein wissenschaftlicher Nachlass auf seinen testamentarischen Wunsch hin verbrannt wurde. Mit den Briefen wenden sich hochrangige Kollegen und Würdenträger an ihn, aber auch Krankenschwestern, die von Ihren Erfahrungen berichten, z. B. während des Ersten Weltkriegs. Es gibt zudem zauberhafte Tonaufnahmen seiner Haushälterin Käthchen Fuchs, die in späteren Jahren recht munter von ihrem nicht immer einfachen Leben im Hause von Herrn und Frau Röntgen berichtet.
Wollen Sie auch digitale, interaktive Formate in die Ausstellung einbinden?

Wir bereiten verschiedene digitale Angebote vor, damit die Besucher das Haus, die Personennetzwerke und die originalen Dokumente kennenlernen können. Mittels Virtual-Reality können sie sich einfach und anschaulich die handgeschriebenen Dokumente erschließen. Die Medien treten aber hinter der Aura der originalen Exponate zurück, sie haben dienende Funktion.
Stichwort Umsetzung - das Geburtshaus soll ja spätestens im ersten Quartal 2019 fertiggestellt sein. Wie sieht der Zeitplan für den Ausstellungsbereich aus?

Er wurden in Abstimmung mit der Deutschen Röntgengesellschaft, dem Deutschen Röntgen-Museum, der Architektin Sophie Welke, die die Sanierung betreut, und der Denkmalpflege wichtige Entscheidungen für die Ausstattung der Räume getroffen, sodass wir jetzt in die finale Ausarbeitung gehen. Auf sie folgen im Frühjahr und Sommer 2018 die technische Produktion und Realisierung. Wir streben eine Eröffnung im Spätherbst 2018 an, aber Qualität geht uns vor Schnelligkeit, da sind wir uns mit allen Beteiligten einig. 